\title{
Signal processing techniques for sustainable cognitive radio communications
}

\author{
Arulmurugan Ramu ${ }^{1} \cdot$ Mu-Yen Chen $^{2} \cdot$ Sri Devi Ravana ${ }^{3} \cdot$ Anandakumar Haldorai $^{4}$
}

Accepted: 9 November 2020/Published online: 18 November 2020

(C) Springer Science+Business Media, LLC, part of Springer Nature 2020

The growing devices and capacity requirements of wireless systems bring increasing demand for RF spectrum. Cognitive radio (CR) system is an emerging concept to increase the spectrum efficiency. CR system aims to enable opportunistic usage of the RF bands that are not occupied by their primary licensed users in spectrum overlay approach. This approach is especially important in signal and image processing, where sets of sensors, large and heterogeneous, provide large amounts of data, usually noisy and corrupted with various sources of interference. From a methodological point of view, cognitive communication is concerned with multi-dimensional and statistical signal processing, especially with problems such as detection, estimation, and optimization. In addition to classical sensing, detection, supervised, reinforcement and learning methods include Bayesian modeling, Markov models, support vector machines, and kernel methods. It spans a broad area of applications, such as military, industrial, medical, transportation and other fields like error control, error detection, adaptive filtering, computer vision, managing data, sensor control, data fusion, blind and semiblind source separation, sparse analysis, brain-computer

Arulmurugan Ramu

arulmurugan@presidencyuniversity.in

Mu-Yen Chen

mychen@nutc.edu.tw

Sri Devi Ravana

sdevi@um.edu.my

Anandakumar Haldorai

anandakumar.h@sece.ac.in

1 Presidency University, Bangalore, Karnataka, India

2 National Taichung University of Science and Technology, Taichung, Taiwan

3 University of Malaya, Kuala Lumpur, Malaysia

4 Sri Eshwar College of Engineering, Coimbatore, Tamil Nadu, India interfaces, signal processing and radio communication. The intelligent system for the cognitive communication is a collection of intelligent terminals with signal processing and mobile capabilities. Among them, each intelligent terminal carries out signal transmission and distributed processing with other intelligent terminals. However, most existing communication architectures, including their signal processing protocols and control algorithms, are designed for centralized networks by default.

This issue aims to gather latest research and development achievements in this area and to promote their applications in all important fields with society needs. This issue features eight selected papers with high quality. The first article, "Salp Swarm Algorithm and Phasor Measurement Unit Based Hybrid Robust Neural Network Model for Online Monitoring of Voltage Stability", presents the online monitoring of voltage stability method. The proposed model is based on Salp Swarm Algorithm based Artificial Neural Network (SSA-ANN). The prime considerations in this model is the use of real time data. The computation time is reduced amongst all the models for all the test cases considered for estimating the VSMI.

The second article, "Efficient Traffic Control and Lifetime Maximization in Mobile Ad hoc Network by Using PSO-BAT Optimization”, proposed hybrid Meta heuristic methodology of dynamic mobile ad hoc network focuses on the mobile node energy level and lifetime maximization with the use of velocity estimation and fitness value calculations of PSO-BAT optimization algorithm. The proposed heuristic algorithm enhances the secured routing process of mobile nodes in dynamic mobile network with denial of service mitigation algorithm in a reliable method.

The third article, "The performance analysis of dualinverter three phase fed induction motor with open-end winding using various PWM schemes", proposed to investigate the performance of various PWM schemes for two inverters fed a three-phase induction motor. The PWM schemes are developed the voltage references and 
modulated using the same or different PWM scheme with simple carrier based PWM method proposed and executed.

The fourth article, "Reliable Service Availability and Access Control Method for Cloud Assisted IoT Communications", the reliable service method availability and access control is discussed to improve the resource allocation and request processing efficiency of Cloud-IoT. This method operates in cloud and gateway layer for resource allocation and request processing respectively. Using a reliable gateway selection method, the requests are processed in a timely manner preventing backlogs and failures. The augmented learning process classifies requests based on time overlap to reduce the backlogs and delay in communication. In the proposed methods, the fore-mentioned processes are augmented to improve the overall performance of cloud assisted IoT platforms.

The fifth article, "Efficient Pre-authentication Scheme for Inter-ASN Handover in High Mobility MANET", proposed a new routing protocol to handle the displacement and direction factors in ad-hoc networks. The proposed Modified EAP based Pre-authentication scheme using Improved ElGamal (MEPIE) overcomes the MITM, replay, DoS and impersonation attacks in MANETs. The proposed mechanism based on the improved ElGamal addresses the inadequacies of ElGamal digital signatures and yields better results.

The sixth article, "Unified Power Quality Conditioner with Reduced Switch Topology for Distributed Networks", In this paper Unified Power Quality Conditioner designed and developed with reduced number of switches and it is controlled by SRF based Carrier Double Zero Sequence Signal Modulation technique. The proposed UPQC system is designed with 10 switches, which provide better performance compared to conventional 12 switch UPQC system. It is implemented to reduce the various power quality issues like minimization of voltage sag and current ripples, harmonics reduction in the input voltage and reduced Total Harmonic Distortion.

The seventh work titled, "False Alarm Detection Using Dynamic Threshold in Medical Wireless Sensor Networks", presents a dynamic threshold algorithm to detect the sensor anomaly to differentiate false and alarms and this system presented for healthcare application in WSN. The error value is calculated using a dynamic threshold which can identify false and true alarm using a majority voting system. The proposed system shows the detection ration is high and false positive rate is lower which conclude that this new approach is particularly useful in WSN application such as health monitoring system and it will be competitive with others.

The eighth article "Design and Development of Planar Antenna Array for MIMO Application" has proposed a Design for implementing an innovative Two T-shaped planar antenna on behalf of ISM band for the MIMO application. The antenna is designed with the Taconic RF35 substrate having a dielectric constant of value 3.5 , while the method of feeding coaxial probe has been employed as antenna feed. The proposed antenna's performance was examined, and it was found that the proposed scheme has better performance compared to the existing schemes.

Our sincere thanks to the Editor-in-Chief, Dr. Imrich Chlamtac and Eliška Vlčková, Managing Editor, European Alliance for Innovation (EAI) for their continuous support and guidance during the entire process. We also extend our sincere thanks and grateful to the reviewers for their efforts in reviewing the manuscripts.

Publisher's Note Springer Nature remains neutral with regard to jurisdictional claims in published maps and institutional affiliations.

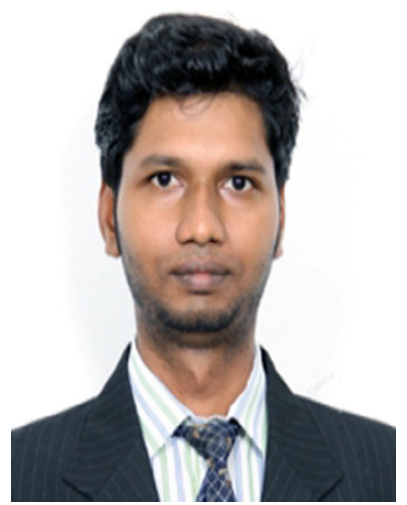

Dr. Arulmurugan Ramu is a Professor, Presidency University, Bangalore, India. His research focuses on the automatic interpretation of images and related problems in machine learning and optimization. His main research interest is in vision, particularly high-level visual recognition. $\mathrm{He}$ has authored more than 35 papers in major computer vision and machine learning conferences and journals. He is the recipient of Ph.D. degrees in Information and Communication Engineering from the Anna University at Chennai, M.Tech. in Information Technology Anna University of Technology and B. Tech degree in Information Technology. He is guided many Ph.D. research scholar under the area of Image Processing using Machine Learning. He is an Associate Editor of Inderscience IJISC journal. He is awarded as Best Young Faculty Award 2018 and nominated for Best Young Researcher Award (Male) by International Academic and Research Excellence Awards (IARE2019).

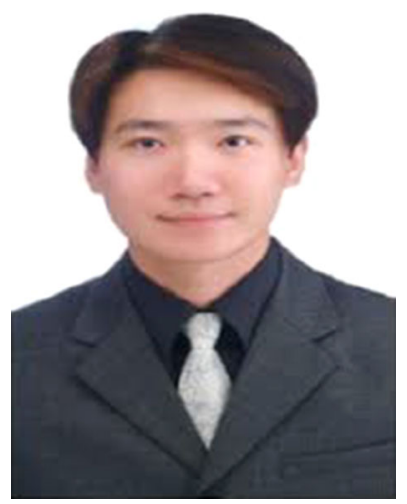

Dr. Mu-Yen Chen is a Professor of Information Management at National Taichung University of Science and Technology, Taiwan. He received his Ph.D. from Information Management from National Chiao-Tung University in Taiwan. His current research interests include artificial intelligent, soft computing, bio-inspired computing, data mining, deep learning, context-awareness, machine learning, and financial engineering, with more than 100 publications in these areas. He has co-edited 12 special issues in International Journals (e.g. Computers in Human Behavior, Applied Soft Computing, Soft Computing, Information Fusion, Neurocomputing, Journal of Medical and Biological Engineering, The Electronic Library, Library High 
Tech). He has served as Editor in Chief and Associate Editor of international journals [e.g. International Journal of Big Data and Analytics in Healthcare, IEEE Access, Human-centric Computing and Information Sciences, Journal of Information Processing Systems, International Journal of Social and Humanistic Computing] while he is an editorial board member on several SCI journals.

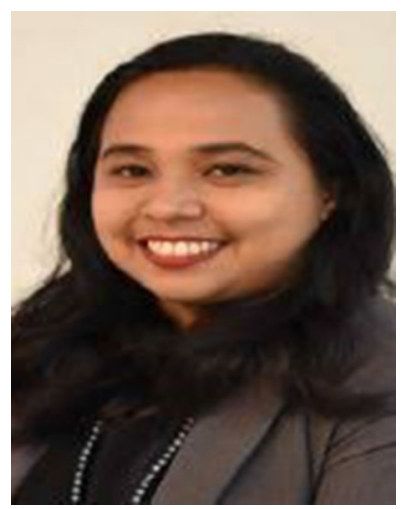

Dr. Sri Devi Ravana received the Bachelor's degree in information technology from the National University of Malaysia, in 2000, the Master's degree in software engineering from the University of Malaya, Malaysia, in 2001, and the Ph.D. degree from the Department of Computer Science and Software Engineering, The University of Melbourne, Australia, in 2012. She is currently an Associate Professor at the Department of Information Systems, University of Malaya. Her research interests include information retrieval heuristics, text indexing, data analytics, and data mining. She received a couple of best paper awards in international conferences within the area of information retrieval.

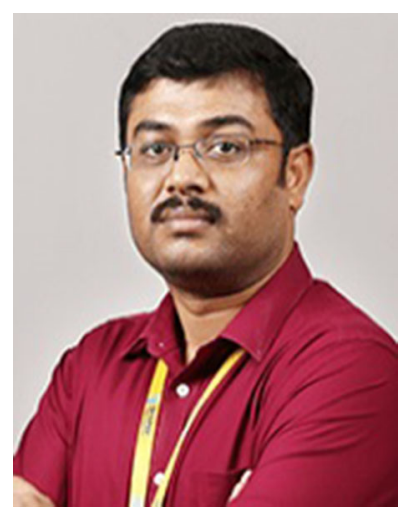

Dr. Anandakumar Haldorai Professor (Associate) and Research Head in Department of Computer Science and Engineering, Sri Eshwar College of Engineering, Coimbatore, Tamil Nadu, India. He has received his Master's in Software Engineering from PSG College of Technology, Coimbatore, $\mathrm{PhD}$ in Information and Communication Engineering from PSG College of Technology under, Anna University, Chennai. His research areas include Cognitive Radio Networks, Mobile Communications and Networking Protocols. He has authored more than 118 research papers in reputed International Journals and IEEE, Springer Conferences. He has authored 11 books and many book chapters with reputed publishers such as Springer and IGI. He has served as Editor in Chief in Keai - Elsevier IJIN and reviewer for IEEE, IET, Springer, Inderscience and Elsevier journals. He is also the guest editor of many journals with Wiley, Springer, Elsevier, Inderscience, etc. He has been the General chair, Session Chair, and Panelist in several conferences. He is a senior member of IEEE, MIET, MACM and Fellow member of EAI research group. 